\title{
Hemicrania continua unresponsive or partially responsive to indomethacin: does it exist? A diagnostic and therapeutic dilemma
}

\author{
Sanjay Prakash · Nilima D. Shah · \\ Roopal J. Bhanvadia
}

Received: 19 October 2008/Accepted: 1 December 2008/Published online: 19 December 2008

(C) Springer-Verlag 2008

\begin{abstract}
Hemicrania continua (HC) is a primary headache disorder characterized by a continuous, unilateral headache that varies in intensity, waxing and waning without disappearing completely. Ipsilateral cranial autonomic features and response to indomethacin are essential features for the diagnosis of HC. We hereby, describe three patients with the clinical phenotypes of $\mathrm{HC}$ in whom response to indomethacin was either incomplete or not sustained. We also review the literature especially for the presence of indomethacin response and ipsilateral cranial autonomic features.
\end{abstract}

Keywords Hemicrania continua $\cdot$ Indomethacin

\section{Introduction}

Hemicrania continua (HC) is an uncommon primary headache syndrome. The syndrome HC presents three characteristics: (1) strictly unilateral continuous pain (moderate, fluctuating), (2) at least one ipsilateral cranial autonomic feature, and (3) complete response to therapeutic doses of indomethacin [1]. However, as $\mathrm{HC}$ is a

S. Prakash $\cdot$ R. J. Bhanvadia

Department of Neurology, Medical College, SSG Hospital, Baroda, Gujarat 390001, India

N. D. Shah

Department of Psychiatry, Medical College, SSG Hospital, Baroda, Gujarat 390001, India

S. Prakash $(\bowtie)$

O-19, Doctor's Quarter, Jail Road, Baroda,

Gujarat 390001, India

e-mail: drprakashs@yahoo.co.in relatively recently described entity, its natural history is still being determined. Knowledge regarding $\mathrm{HC}$ has rapidly changed over years. Even the diagnostic criteria of HC have been repeatedly modified and revised over the last 10 years [1-3]. All the three characteristic features are essential to satisfy the International Headache Society (IHS) criteria for HC. However, the criteria proposed by other authors are more accommodating [2]. If we strictly adhere to the IHS criteria for HC, many cases reported earlier may not be labeled as HC. We here, describe three patients with the clinical features of $\mathrm{HC}$ in whom response to indomethacin was either incomplete or not sustained.

\section{Case reports}

\section{Case 1}

A 30-year-old man presented with a 4-month history of continuous right-sided headache. The pain was maximal in the right supraorbital and retro-orbital areas radiating to the temporoparietal and maxillary regions. The pain was described as a constant ache of moderate severity with superimposed exacerbations of severe to excruciating pulsating pain lasting 2-60 min. Exacerbations occurred 3-20 times in a day, and were associated with ipsilateral conjunctival injections, lacrimation and rhinorrhea in about $50 \%$ of the attacks. The patient never developed aura, nausea, photophobia, phonophobia or restlessness (agitation) during the exacerbations. Nocturnal attacks were common. There were no precipitating or aggravating factors. The patient had no prior history of headache or head trauma. Family history for headache was negative. Past treatments with paracetamol, ibuprofen, tramadol, amitriptylin, and propranolol were without benefit. 
Physical examinations and investigations [including magnetic resonance imaging (MRI) brain] were normal. Diagnosis of $\mathrm{HC}$ was made. He showed complete response to indomethacin at the dose of $75 \mathrm{mg}$ tid. The patient had never felt such type of improvement (either spontaneously or with any drugs) since the beginning of his clinical presentation. However, response persisted only for 2 days and the headache recurred with the same intensity and frequency. Indomethacin was gradually increased up to $150 \mathrm{mg}$ tid. However, the patient did not feel any relief in his symptoms. Further trials with topiramate and gabapentine were without any benefit. The patient lost to follow-up after 6 weeks.

\section{Case 2}

A 46-year-old female presented with a 5-year history of continuous right-sided headache with superimposed exacerbations. The continuous pain was dull, of moderate severity, and maximal in the retro-orbital and infraorbital areas radiating to the entire hemicranium (including teeth and gums). The exacerbations, described as throbbing and/ or stabbing type of pain, were associated with ipsilateral cranial autonomic features (especially ptosis and conjunctival injection) in about one-third of the attacks. Exacerbations occurred 10-20 times daily, lasting $10 \mathrm{~min}$ to $2 \mathrm{~h}$.

Prior treatments with propranolol, aspirin, ibuprofen, topiramate, sodium valproate, amitryptiline, gabapentine were without benefit. Dental extraction was done twice, suspecting that pain was of dental origin. The patient was extensively investigated for 5 years. She got done a MRI of the brain and orbit two times, which revealed no abnormality. General and neurological examinations were normal. A diagnosis of $\mathrm{HC}$ was made and indomethacin was started at a dose of $25 \mathrm{mg}$ tid.

No response was noted at this dose. It was gradually increased to $100 \mathrm{mg}$ tid. With this dose, she noted marked (but incomplete) improvement in 7 days. Her background headache subsided completely. The frequency of attacks decreased to 1-3 per day. The intensity and duration of attacks also reduced markedly. Further, increase of the dose (up to $150 \mathrm{mg}$ tid) did not provide any additional benefit. The patient developed pain abdomen after about 15 days and indomethacin was stopped. However, symptoms recurred on the same day with the same intensity, duration, and frequency. Indomethacin was reinstituted after 2 weeks with gastric protection. The dose was gradually increased up to $100 \mathrm{mg}$ tid. The patient again showed partial improvement. There was only a mild background headache (at times the background headache also disappeared). In next 2 months of follow-up, she had 1-2 episodes of exacerbation in a day.
Case 3

A 29-year-old female presented with a 6 months history of continuous left-sided headache. The pain was described as a steady ache of mild to moderate severity with superimposed exacerbations of throbbing pain, recurring 1-3 times daily. Exacerbations lasted from $10 \mathrm{~min}$ to $2 \mathrm{~h}$ and were associated with ipsilateral conjunctival injection, lacrimation, nausea, photophobia, and phonophobia. The pain was maximal in the left orbit radiating to the temporoparietal and maxillary areas. There were occasional nocturnal attacks. No triggering factors were noted. Family history for headache and history of head trauma were negative. Prior treatments with propranolol, amitriptyline, naproxen, and paracetamol were ineffective. Neurological examination, routine hematological, biochemical examinations, and MRI of the brain were normal. Treatment with indomethacin $25 \mathrm{mg}$ tid was ineffective. Gradually increasing the dosage to $100 \mathrm{mg}$ tid produced incomplete improvement. Her background headache reduced in intensity (However, it persisted in a continuous pattern). The frequency of exacerbations reduced to 1-2 episodes in a week. However, the intensity of exacerbations (severe, disturbing routine activities) and the duration (10 min to few hours) were the same, as she had been suffering previously before the introduction of indomethacin. After 6 weeks of introduction of indomethacin (100 mg tid), we gave IV MPS $(500 \mathrm{mg})$ daily in normal saline for 3 days with gastric protection. We asked the patient to continue indomethacin in the same dose (100 mg tid). The background headache subsided completely on the first day. In the next 3 months, she had no background headache, nor any exacerbation. Hence, we tapered the dose of indomethacin. Tapering further (below $50 \mathrm{mg}$ tid) resulted in the reappearance of the background headache and the exacerbations.

\section{Discussion}

The clinical features and indomethacin-responsiveness of $\mathrm{HC}$ were first described by Medina and Diamond. The term "hemicrania continua" was coined by Sjaastad and Spierings [4], and considered it as an indomethacin-responsive headache.

According to the present diagnostic (IHS) criteria except the one stating that a complete and persistent response to therapeutic doses of indomethacin is a must, all three of our cases presented phenotypically as HC. The clinical phenotype of $\mathrm{HC}$ overlaps with that of migraine and trigeminal autonomic cephalalgias (TACs). The frequencies of exacerbations match with paroxysmal hemicrania $(\mathrm{PH})$ in cases 1 and 2 , and with cluster headache $(\mathrm{CH})$ in patient 3 . The duration and frequency of exacerbations are not defined in 
the $\mathrm{HC}$ criteria. However, the frequency may vary from 20 attacks per day to few attacks in a week [5]. Therefore, the background headache is the most important feature to differentiate HC from TACs (as paroxysmal hemicrania and even few cases of cluster headache show response to indomethacin) [6]. Although, presence of inter ictal (background) pain in the patients with $\mathrm{PH}$ and $\mathrm{CH}$ may further complicate the diagnosis of HC [7]. Presence of moderate background pain is the most important point to differentiate $\mathrm{HC}$ with $\mathrm{PH}$ and $\mathrm{CH}$ [5, 8]. Newman et al. [5] commented "If the patient or the doctor focuses on the painful exacerbations with autonomic features and fails to consider the continuous, moderate background pain, HC may be mistaken for cluster headache", and probably PH. The background headache was moderate in all the three patients.

None of our patients fulfilled the criteria of migraine. Frequent attacks of migraine (of smaller duration) in a day were not in favor of migraine. Other differential diagnosis of $\mathrm{HC}$ may include supraorbital neuralgia, cervicogenic headache, unilateral tension type headache, atypical facial pain, and temporomandibular joint pain [9]. However, continuous background pain and periodic exacerbations especially in retro-orbital areas, and autonomic features are against the diagnosis of almost all the above-mentioned differential diagnosis. Case 1 showed complete response to indomethacin, but it persisted only for 2 days. Case 2 showed partial response even at the dose of $150 \mathrm{mg}$ tid. Case 3 showed incomplete response at the dose of $100 \mathrm{mg}$ tid. However, a 3 days course of IV MPS provided a complete response. Recently, two patients of HC were reported who showed complete response to IV MPS (without concomitant use of indomethacin) [10]. However, case 3 was also receiving indomethacin during the administration of IV MPS. The temporal relation of the disappearance of headache and the administration of IV MPS suggests that MPS was pivotal in the disappearance of headache. However, recurrence of headache after 3 months on withdrawal of indomethacin suggests that the headache was responsive to indomethacin. Probably, IV MPS facilitated the effect of indomethacin in the early part of the treatment.

The variability in time interval between indomethacin administration and response (few hours to 10 days) and variability in indomethacin requirement with time may be the causes of unresponsiveness in few patients of indomethacin unresponsive HC [11]. There is suggestion to give indomethacin parenterally (INDOTEST) to avoid such problem [12]. However, injectable indomethacin is not available in every country. The maximum dose and duration of indomethacin in our patients were: $150 \mathrm{mg}$ tid for 6 weeks (case 1), $150 \mathrm{mg}$ tid for 2 weeks (case 2), and $100 \mathrm{mg}$ tid for 6 weeks (case 3). Therefore, the possibility of use of suboptimal dose or the possibility of delayed response does not exist in our patients.

Sjaastad and Spierings [4] in their original article of HC comment, "cases unresponsive to indomethacin but otherwise corresponding entirely to the present individuals may well be discovered in the future". However, they failed to find such patients and later on made a comment, "...a continuous, unilateral headache without response to indomethacin is not hemicrania continua" [13]. However, many other authors accepted the possibility of occurrence of indomethacin-resistant $\mathrm{HC}$ and, diagnostic criteria have been proposed that accommodate both indomethacinresponsive and indomethacin-resistant patients who fit the clinical phenotype [2, 3, 9]. Pareja et al. [9] proposed the term "hemicrania generis incerti" for the indomethacinresistant HC. Goadsby and Lipton [11] suggested the term hemicrania continua (possible) for the patients who fulfilled criteria except the indomethacin response. A complete and persistent response to indomethacin was included as a pre-requisite criterion for the diagnosis of $\mathrm{HC}$ by the classification criteria of the IHS in 2004 [1]. Many cases (at least 13) have been reported (before 2004), where typical cases of HC did not respond to indomethacin [1418]. Kuritzky [15] reported four patients who otherwise met clinical criteria for $\mathrm{HC}$ yet failed to respond to treatment with indomethacin. However, the maximum dosage used was $100 \mathrm{mg}$ of indomethacin daily, and the possibility remains that higher doses may have provided benefit. Pascual [14] reported another patient unresponsive to $225 \mathrm{mg} /$ day of indomethacin. Wheeler [16] reported six patients of $\mathrm{HC}$ who did not show response to indomethacin. Besides these, there are many other cases, where response to indomethacin was not complete or sustained [17, 18]. However, there is no case report of indomethacin-resistant HC (or HC like headache) in the literature after 2004. Nevertheless, we found at least two cases in the literature, where response to indomethacin was not complete or persistent $[19,20]$. The sudden scarcity of the indomethacinresistant $\mathrm{HC}$ in the literature (after 2004) may be because of under (or no) reporting of such type of headache, as it is difficult to classify $\mathrm{HC}$ like headache unresponsive to indomethacin. Newman et al. [21] wrote, "We too have observed patients with headaches clinically indistinguishable from $\mathrm{HC}$ that do not respond to maximum doses of indomethacin. We do not know how to classify these unusual patients. They may be a treatment-refractory subgroup of HC patients". However, on review of the literature we did not find any such patients (indomethacinresistant HC) by Newman in the literature. This observation further suggests about under (or no) reporting of such type of headache. Response to indomethacin was an inclusion criterion in the observation or studies even before 2004 [22, 23]. Therefore, the possibility of the under 
reporting of $\mathrm{HC}$ like headache exists even before the publication of IHS classification.

As response to indomethacin is an essential feature for the diagnosis of HC, physicians do not take trials of other drugs (especially in the early part of the treatment). Although, various drugs are reported effective (usually in isolated cases) in the patients with $\mathrm{HC}$, no drug (other than indomethacin) is found consistently effective [24]. This creates a problem for the patient (and the treating physician). Case 1 lost to follow-up, as we could not provide any relief to him.

At least one of the autonomic features (ipsilateral to the side of pain) during the exacerbations is one of the essential features in the IHS diagnostic criteria. According to this criterion, many of the patients described in the literature cannot be labeled as (at least definite) HC. Nine patients (out of the 34) (26\%) of Peres et al. [23] series did not show any autonomic features. The authors reviewed the 41 other cases in literature, and noted the absence of autonomic features in 15 cases $(37 \%$ ). Three patients (out of 10) of Bigal et al. [25] series did not have any autonomic features. This observation raises the question that how to classify a patient with HC like headache who shows a response to indomethacin, but does not have autonomic features. Can we classify these cases as HC? Again, we did not find any single case report of $\mathrm{HC}$ without autonomic features in the literature after publication of the IHS classification (for HC). This again suggests under (or no) reporting of such type of cases, as it is difficult to classify such type of patients according to present IHS classification.

Our case reports and review of the literature suggest that there is a subset of patients with clinical phenotype of $\mathrm{HC}$, who do not satisfy all features of the IHS diagnostic criteria. Bigal et al. [25] comment “...it seems somewhat exaggerate to consider that $\mathrm{HC}$ not responsive to indomethacin does not exist". We suggest a more inclusive set of criteria, as proposed by Goadsby and Lipton [2], where either indomethacin-responsiveness or the presence of one cranial autonomic feature is sufficient to make the diagnosis of HC. Hemicrania continua (possible), as suggested by Goadsby and Lipton [11], may be another way to classify such types of patients. The remarkable response of indomethacin in patients with $\mathrm{HC}$ suggests that indomethacin probably act on a very specific point in $\mathrm{HC}$ pathophysiology. Thus, indomethacin-resistant $\mathrm{HC}$ mean a headache that lacks this indomethacin specific site of action. Therefore, the possibility of an independent disorder (clinically similar to HC) cannot be ruled out. The study on pathophysiology of both types of headache (i.e. $\mathrm{HC}$ and $\mathrm{HC}$ like headaches resistant to indomethacin) will help in solving the problem. However, until the underlying pathophysiology of $\mathrm{HC}$ is understood, it will be better to use more accommodating type of criteria for clinical purpose (as proposed by Bigal et al.) [26].

Conflict of interest None.

\section{References}

1. Headache Classification Subcommittee of the International Headache Society (2004) The international classification of headache disorders, 2nd edn. Cephalalgia 24(Suppl 1):1-160

2. Goadsby PJ, Lipton RB (1997) A review of paroxysmal hemicranias, SUNCT syndrome and other short-lasting headaches with autonomic feature, including new cases. Brain 120:193-209

3. Pareja JA, Vincent M, Antonaci F, Sjaastad O (2001) Hemicrania continua: diagnostic criteria and nosological status. Cephalalgia 21:874-877

4. Sjaastad O, Spierings EL (1984) Hemicrania continua: another headache absolutely responsive to indomethacin. Cephalalgia 4:65-70

5. Newman LC, Lipton RB, Solomon S (1994) Hemicrania continua: ten new cases and a review of the literature. Neurology 44:2111-2114

6. Prakash S, Dholakia SY, Shah KA (2008) A patient with chronic cluster headache responsive to high-dose indomethacin: is there an overlap with chronic paroxysmal hemicrania? Cephalalgia 28:778-781

7. Goadsby PJ, Cittadini E, Burns B, Cohen AS (2008) Trigeminal autonomic cephalalgias: diagnostic and therapeutic developments. Curr Opin Neurol 21:323-330

8. Cittadini E, Matharu MS, Goadsby PJ (2008) Paroxysmal hemicrania: a prospective clinical study of 31 cases. Brain 131:11421155

9. Pareja JA, Antonaci F, Vincent M (2001) The hemicrania continua diagnosis. Cephalalgia 21:940-946

10. Prakash S Brahmbhatt KJ, Chawda NT, Tandon N (2008) Hemicrania continua responsive to intravenous methyl prednisolone. Headache. doi: 10.1111/j.1526-4610.2008.01269.X

11. Goadsby PJ, Lipton RB (2002) The hemicrania continua diagnosis. Cephalalgia 22:563-565

12. Antonaci F, Parja JA, Caminero AB, Sjaastad O (1998) Chronic paroxysmal hemicrania and hemicrania continua. parenteral indomethacin: the 'indotest'. Headache 38:122-128

13. Spierings LH, Sjaastad O, F.P. Peres M, Silberstein SD, Young WB, Rozen TD (2002) Hemicrania continua is not that rare. Neurology 59:476-477

14. Pascual J (1995) Hemicrania continua. Neurology 45:2302-2303

15. Kuritzky A (1992) Indomethacin-resistant hemicraniacontinua. Cephalalgia 12:57-59

16. Wheeler S (2000) Clinical spectrum of hemicrania continua. Neurology 54:A422 abstract

17. Wheeler SD (2002) Hemicrania continua in African Americans. J Natl Med Assoc 94(10):901-907

18. Mariano da Silva H, Alcantara MC, Bordini CA, Speciali JG (2002) Strictly unilateral headache reminiscent of hemicrania continua resistant to indomethacin but responsive to gabapentin. Cephalalgia 22:409-410

19. Schwedt TJ, Dodick DW, Trentman TL, Zimmerman RS (2006) Occipital nerve stimulation for chronic cluster headache and hemicrania continua: pain relief and persistence of autonomic features. Cephalalgia 26:1025-1027

20. Rajabally YA, Jacob S (2005) Hemicrania continua responsive to verapamil. Headache 45:1082-1083

21. Newman LC, Solomon S, Lipton RB (1995) Reply from author: hemicrania continua. Neurology 45:2303 
22. Bordini C, Antonaci F, Stovner LJ, Schrader H, Sjaastad O (1991) 'Hemicrania continua': a clinical review. Headache $31: 20-26$

23. Peres MF, Silberstein SD, Nahimas S, Shechter AL, Youssef I, Rozen TD, Young WB (2001) Hemicrania continua is not that rare. Neurology 57(6):948-951

24. Matharu MS, Boes CJ, Goadsby PJ (2003) Management of trigeminal autonomic cephalgias and hemicrania continua. Drugs 63(16):1637-1677
25. Bigal ME, Tepper SJ, Sheftell FD, Rapoport AM (2002) Hemicrania continua: a report of ten cases. Arq Neuropsiqiatr 60:695698

26. Bigal ME, Tepper SJ, Rapoport AM, Sheftell FD (2002) Hemicrania continua: comparison between two different classification systems. Cephalalgia 22:242-245 